REVISIÓN

Rev Colombiana Cienc Anim 2016; 8(Supl):391-399.

\title{
La etología como herramienta para la conservación de fauna silvestre
}

\section{Ecology as a tool for wildlife conservation}

\author{
SAMPEDRO MARÍN, ALCIDES ${ }^{1}$ Ph.D.
}

${ }^{1}$ Universidad de Sucre. Grupo de Investigación en Biodiversidad Tropical. Sincelejo, Sucre.

\section{Key words:}

Ethology;

evolution;

adaptations;

behavioral ecology;

conservation;

biodiversity.

\section{Abstract}

Se explican los orígenes de la etología como disciplina y que constituye una prueba de la teoría darwiniana sobre la acción de la selección natural dando lugar a las estrategias adaptativas que permiten la supervivencia de los organismos vivos. Se destaca el surgimiento de la ecología de la conducta como una herramienta importante para la conservación de la diversidad biológica y se explican sus premisas, así como varios ejemplos de conductas que afectan el tamaño efectivo de las poblaciones e impactos antropogénicos sobre diversas conductas. Finalmente, se ejemplifica el uso de la ecología del comportamiento como un indicador del estado de los ecosistemas y de las especies y el desarrollo de la educación ambiental.

\section{Palabras Clave:}

Etología; evolución; adaptaciones; ecología de la conducta; conservación; diversidad biológica.

\section{Resumen}

The origins of ethology as a discipline are explained and is a proof of the Darwinian theory of the action of natural selection leading to the adaptive strategies that allow survival of living organisms. The emergence of behavioral ecology stands out as an important tool for the conservation of biological diversity. Its premises are explained, as well as several examples of behavior that affect the effective size of populations and anthropogenic impacts on various behaviors. Finally, the use of behavioral ecology as an indicator of the state of ecosystems and species and to develop environmental education is exemplified. 


\section{Introducción}

La conducta animal se ha estudiado desde sus inicios a partir de trabajos en zoología y psicología experimental y siguió esas dos vertientes a través de la etología, que hace estudios biológicos de la conducta instintiva y la zoopsicología que estudia los procesos nerviosos superiores, especialmente el aprendizaje.

La etología se ha conceptualizado de variadas formas desde su aparición como disciplina y curiosamente siempre en términos algo ambiguos, quizás por la amplitud del conocimiento que abarca. Así, TINBERGEN (1951) la definió como un estudio objetivo del comportamiento y SOLER (2009) le da un enfoque adaptacionista al plantear que se trata de la ecología del comportamiento. La mayoría de los autores coinciden en que el comportamiento animal se evidencia a través de "determinadas actividades que se realizan con secuencia en el tiempo y que se manifiestan, en la mayoría de las ocasiones, como movimientos musculares y cambios en la actividad glandular (BEROVIDES, 1988).

Desde pequeños somos testigos de innumerables ejemplos del comportamiento de los animales, sobre todo porque tenemos mascotas, asistimos al zoológico y fincas, donde constantemente aparecen tales actividades, casi siempre relacionadas con la alimentación, la reproducción, la agresividad, entre otras. Es común escuchar las difíciles preguntas de los niños a sus padres acerca de variados temas, como por ejemplo: ¿Por qué la vaca está lamiendo al ternero que acaba de nacer?, ¿Por qué ese pajarito abre las alas y canta dando vueltas alrededor del otro?, ¿Qué le ocurre a esa lagartija?, estaba verde y se puso casi negra. Las soluciones a esas y otras numerosas interrogantes pueden hallarse en la etología, una disciplina que trasciende hasta nuestros días, desde el siglo XIX, convirtiéndose en una importante ciencia que ha permitido a nuestra especie importantes logros en el manejo de la fauna silvestre y doméstica (SAMPEDRO \& CABEZA, 2009).

Se considera el padre de la etología al zoólogo norteamericano Charles Otis Whitman, quien vivió en el siglo XIX y principios del XX. Sin embargo, quienes más desarrollaron esta disciplina posteriormente fueron el austriaco Konrad Z. Lorenz (1903-1989), a quien se debe la descripción de la impronta animal (CAMPBELL, 1975); y NikolaasTinbergen (1907-1988), nacido en Los Países Bajos, quien reconociólos estímulos como señalesque originan las conductas de los animales, lo que según él se encuentra coordinado por el sistema nervioso (KRUUK, 2003). Ambos investigadores, junto al también zoólogo austriaco Karl R. von Frisch (1886-1982), recibieron el premio Nobel en Fisiología o Medicina en 1973.

Los etólogos actuales incursionan en los orígenes de la conducta animal y por supuesto en su valor adaptativo (CARRANZA, 2016), tema que destaca el papel de la selección natural en el desarrollo de diferentes conductas que permiten la supervivencia de la fauna silvestre y doméstica. También se le da importancia a la etología como una herramienta para la conservación de la fauna silvestre (BEISSINGER, 1997; RUBENSTEIN, 1998; CASSINI, 1999; SAMPEDRO y CABEZAS, 2009), debido a la situación por la que atraviesa la diversidad biológica por los cambios globales del clima, la extinción de especies y el deterioro evidente del ambiente en todo el planeta.Es una disciplina que se encuentra enmarcada en la teoría Darwinista de la evolución.

En esta revisión pretendo llamar la atención, sobre todo de los investigadores jóvenes y los futuros profesionales, para que asuman el reto de profundizar en la conducta animal como una ciencia que permite explicarnos la vida, corroborar los principios neo darwinistas sobre la evolución de los organismos y contribuir activamente a su conservación.

\section{Las 4 preguntas de Tinbergen}

Según TINBERGEN (1963) en Biología se puede responder al ¿por qué? de variadas maneras. Se le conoce como las 4 preguntas de Tinbergen. KREBS \& DAVIES (1993) exponen con un ejemplo el significado del planteamiento de Tinbergen.

Si preguntamos por qué el estornino Sturnus vulgaris canta en primavera podemos responder como sigue:

1.En términos de valor de supervivencia o función. Los estorninos cantan para atraer pareja para el apareamiento. 
2. En términos de causalidad. Cantan porque el aumento de la duración del día desencadena cambios en los niveles corporales de hormonas, o debido a la forma en que el aire fluye a través de la siringe y establece las vibraciones de la membrana.

Estas son respuestas acerca de los factores externos e internos que causan el canto de los estorninos en primavera.

3. En términos de desarrollo. Cantan porque ellos han aprendido esos cantos de sus padres y otros congéneres.

4.En términos de historia evolutiva. Esta respuesta se referiría a cómo el canto ha evolucionado desde sus ancestros hasta la actualidad. Las aves vivientes más primitivas hacen simples sonidos, luego es razonable asumir que los cantos complejos de los estorninos y otras aves han evolucionado a partir de simples llamados ancestrales.

Es importante para los investigadores distinguir estas variadas formas de responder una pregunta en Biología, porque de lo contrario puede perderse el tiempo en discusiones estériles, ya que todos pueden tener razón.

\section{Conducta y evolución}

Según LORENZ et al. (1981), muchos años antes Whitman había enunciado que "Tanto los órganos como los instintos se deben estudiar desde el punto de vista de su origen filético". Estos autores en su trabajo utilizan el método comparativo desarrollado por DARWIN en EI Origen de las Especies (1859) en cuanto a los cambios morfológicos y fisiológicos de grupos relacionados, para tratar de descubrir la evolución de la conducta. Todo biólogo, plantean, para conocer por qué la oreja se encuentra detrás de la mandíbula tiene que mirar otros grupos relacionados y estudiar los cambios que se observan en al aparato auditivo, lo mismo sucede cuando se desea determinar la presencia de la mano en lugar del ala de las aves o de las patas con garras de los reptiles.

El papel de la selección natural actuando sobre caracteres morfológicos y fisiológicos también se hizo evidente sobre los caracteres conductuales. Para ello Lorenz expuso ejemplos de sus observaciones personales cuando afirmaba que la manera en que los perros y las aves se rascaban utilizando ambos grupos las patas traseras, los primeros cruzándola por encima de las patas delanteras y los segundos por encima del ala, solo puede explicarse planteando que ambos grupos tienen un origen común.Actualmente la mayoría de los especialistas reconoce el hecho de que, al igual que los caracteres morfológicos y fisiológicos, los caracteres conductuales que aparecen en las diferentes especies son el producto de mutaciones al azar que se mantienen o eliminan por acción de la selección natural. CARRANZA (2016) concentra su atención en cómo el comportamiento ha sido producido por la selección natural. Los organismos vivos resuelven los problemas ambientales que enfrentan, es decir las presiones selectivas. Lo hacen mediante la reproducción pasando a las próximas generaciones los genes capaces de responder adecuadamente, o sea adaptándose. Entender eso posibilita la comprensión de cómo ha evolucionado el mundo vivo.

Otras corrientes modernas han tratado de explicar que la evolución no ocurre de forma gradual en largos periodos de tiempo. SHAPIRO (2011), responsable del concepto de "ingeniería genética natural" explica cómo se crea novedad en el curso de la evolución biológica y plantea que la evolución ya no debe explicarse en términos de mutaciones casuales seguido de selección natural, porque las células crean con un fin, presionadas por el ambiente, es decir, en las células de los organismos ocurren cambios que no son casuales, sino que tienen como objetivo que sobreviva frente a las presiones ambientales. Tal teoría tiene sus fundamentos en las respuestas rápidas de ciertas bacterias a condiciones hostiles del ambiente, pero, ¿podría ser esta la explicación acerca de la evolución de la compleja conducta de apareamiento de la mayoría de los vertebrados terrestres y acuáticos, o de la conducta social de los himenópteros con su eficiente división de las tareas que garantiza la vida de miles de individuos? ¿Serían estos procesos respuestas rápidas y no graduales?

BEROVIDES (1988) plantea que cuando se tiene un grupo de especies relacionadas, en el que se puede demostrar que unas son más primitivas que las otras mediante criterios morfológicos y fisiológicos, puede apreciarse que las conductas también cambian y casi siempre son más 
complejas en las especies menos primitivas, lo que evidencia el hecho de que la conducta en cuestión ha sufrido una evolución que ha sido causada por los mismos mecanismos que actúan para los caracteres morfológicos y fisiológicos. Este autor expone un ejemplo muy ilustrativo al respecto, sobre la conducta de construcción del nido en las especies del periquito del amor del género Agapornis (Tabla 1).

Tabla 1. Conducta de construcción del nido en las especies del periquito del amor del género Agapornis, según BEROVIDES (1988).

\begin{tabular}{ccc}
\hline $\begin{array}{c}\text { Condición evolutiva } \\
\text { de cada especie }\end{array}$ & Especie & Nido \\
\hline Primitiva & $\begin{array}{c}\text { Periquito de } \\
\text { Madagascar }\end{array}$ & $\begin{array}{c}\text { En hueco, no modelado, suelo } \\
\text { almohadillado con pequeñas tiras }\end{array}$ \\
Intermedia & $\begin{array}{c}\text { Periquito cara } \\
\text { de melocotón }\end{array}$ & $\begin{array}{c}\text { En hueco, modelado, utilizan tiras largas } \\
\text { de corteza y hojas }\end{array}$ \\
Reciente & $\begin{array}{c}\text { Periquito de } \\
\text { Fisher }\end{array}$ & $\begin{array}{c}\text { En hueco, cubierto y complicado, en } \\
\text { forma de túnel, con ramitas y tiras de } \\
\text { corteza y hojas }\end{array}$ \\
\hline
\end{tabular}

Otro mecanismo para demostrar que el comportamiento tiene bases genéticas puede encontrase en un ejercicio de selección artificial que realizó MANNING (1963), quien fue capaz de seleccionar dos velocidades de apareamiento diferente en la mosca de la fruta (Drosophyla melanogaster), por la cría selectiva de individuos que se apareaban rápido y los que se apareaban lento. Estos experimentos de selección casi siempre funcionan y muestran que gran parte de la variación fenotípica que se observa a menudo en las poblaciones tienen una base genética.

Un aspecto importante sobre los patrones de conducta de los animales, es si son adaptativos para ellos como individuos y para su especie como un todo. Si entendemos por "adaptación" toda estructura o función que adecúa al organismo a su ambiente, de tal forma que le permite llevar a cabo todas sus actividades vitales de manera normal y así sobrevivir y reproducirse, es obvio que la inmensa mayoría de patrones conductuales como los relacionados con la alimentación, reproducción, escape de depredadores entre otros, tienen un gran valor adaptativo, ya que ayudan al individuo a sobrevivir y perpetuar la especie.
Luego, en este caso la investigación debe encaminarse hacia la búsqueda de los factores ambientales, a los cuales la especie, "como un todo" ha respondido con las adaptaciones conductuales en estudio. Por ejemplo, la conducta social de muchos primates tiene un claro valor adaptativo, toda vez que se protege a los jóvenes y hembras y además, como los machos mayores son privilegiados por ser los únicos que pueden aparearse con las hembras, se garantiza que a la nueva generación pasen los genes de los individuos más experimentados y mejor adaptados. Sin embargo, los factores ambientales que determinan esta conducta pueden variar mucho. En unos casos puede ser la presión de los depredadores o las condiciones ambientales adversas en cuanto a clima, tipo de vegetación, alimento, entre otras causas. Estos grupos podrían variar mucho su comportamiento en nuevas condiciones ambientales.

En otros casos, la conducta en sí aparentemente no tiene ventaja adaptativa y el problema está en investigar si esto es así, o si su valor adaptativo está enmascarado por otros factores. Por ejemplo, aún se discute por los especialistas si la conducta de juego de muchos mamíferos jóvenes posee algún valor adaptativo, aunque hay consenso acerca de que les permite desarrollar habilidades para su vida adulta en lo relacionado con la caza, el emparejamiento, la lucha o la huída.

Por último, debe quedar claro que una conducta es adaptativa no porque el animal entiende que su conducta es adecuada, sino porque las fuerzas evolutivas han actuado y producido un organismo que sólo puede realizar esta conducta en esa situación dada.

\section{Conducta y conservación}

La riqueza de especies y el endemismo son quizá el patrimonio más importante que tiene Colombia (GONZÁLEZ-ZUARTH, 2015), sin embargo, plantea el autor, "se observa un deterioro ambiental sin precedentes que estágenerando cambios globales, destrucción de ecosistemas,extinción de especies, agotamiento de los recursos naturales,contaminación de agua, suelo, aire y enfermedades emergentes que afectan todas las formas de vida". Los esfuerzos que se lleva 
a cabo para frenar esta situación no parecen ser suficientes porque, según el autor, solo en lo relacionado con la amenaza a especies silvestres, de las 35.476 especies de fauna que viven en Colombia, 395 están amenazadas. Esta situación se explica por el papel preponderante que la política y la economía tienen sobre la conservación de la diversidad biológica y los recursos naturales en la sociedad en que vivimos.Sin embargo, soy del criterio que también influye el escaso conocimiento que aún se tiene sobre qué hacer para lograr una conservación más eficiente.

Una disciplina relativamente nueva en Latinoamérica parece aportar elementos de importancia para apoyar las labores de conservación, es la Ecología de la Conducta. En México se trabaja en ella hace varias décadas ejemplo de ello es la UNAM donde funciona el Laboratorio de Conducta Animal que pertenece al Departamento de Ecología Evolutiva del Instituto de Ecología de esa Universidad mexicana; En Argentina es materia optativa de la Licenciatura en Ciencias Biológicas, Departamento de Ecología Genética y Evolución, Facultad de Ciencias Exactas y Naturales, Universidad de Buenos Aires; En la Universidad Nacional de Educación a Distancia en Colombia, se imparten cursos de Psicobiología donde se tocan aspectos que guardan relación con la ecología del comportamiento, pero aplicados a la especie humana.

No obstante, BERGER et al. (2011) plantearon que los argumentos que permitían enlazar estas disciplinas resultaban aún débiles y esto los llevó a desarrollar un modelo conceptual que consiste en un marco estructurado, jerárquico y parsimonioso que establece un puente entre ambas disciplinas y muestra un objetivo común en el que el campo de la conducta de ahorro puede evolucionar. Los temas básicos del modelo de estos autores son tres: 1) Los impactos antropogénicos directos e indirectos sobre la conducta animal, lo que eventualmente afecta la diversidad biológica, 2) Gestión basada en el comportamiento, es decir, la consideración de la conducta en la práctica de la conservación basada en el comportamiento; 3) Indicadores de comportamiento a otros procesos que son de interés para la conservación. Por supuesto, la naturaleza adaptativa de la conducta constituye la clave de la ecología del comportamiento, ya que la presión selectiva que supone el ambiente será el origen de las respuestas de comportamiento en una población, en un proceso evolutivo que dependerá de la idoneidad de las estrategias particulares bajo las condiciones ambientales reinantes, o sea, el comportamiento de las diferentes especies de fauna silvestre puede explicar sus estrategias a largo plazo frente a los cambios ambientales (FESTA-BIANCHET y APOLLONIO, 2013).

Para GONZÁLEZ-ZUARTH (2015) la ecología de la conducta parte de tres premisas: 1) el principal objetivo de cualquier organismo es perpetuar su linaje a través del tiempo maximizando su adecuación. 2) La supervivencia y el éxito reproductivo de un individuo depende básicamente de las estrategias conductuales que siga cotidianamente, y 3 ) las estrategias más exitosas son aquellas que maximizan la diferencia entre los beneficios y los costos de implementarlas. Como se aprecia de nuevo queda implícito el papel de las estrategias conductuales de los organismos para sobrevivir ante los cambios ambientales.

En varios trabajos se ha destacado la importancia que la ecología del comportamiento puede tener para la solución de problemas prácticos de conservación (CARO, 1998; BLUMSTEIN y FERNÁNDEZ-JURICIC, 2010; GONZÁLEZZUARTH, 2015). Este último autor ha señalado varios ejemplos en su artículo que resultan muy esclarecedores. Los lobos, perros salvajes y otros animales que utilizan la estrategia de grupo para cazar, disminuyen su efectividad para lograrlo cuando por acción del hombre (cacería) tales grupos decrecen drásticamente en su número. De igual forma los venados, ciervos y otros que basan su estrategia adaptativa para sobrevivir en la formación de grupos familiares, al disminuir su número por la acción antropogénica, se ven obligados a aumentar el tiempo de vigilancia $y$, en consecuencia, a disminuir el tiempo que pasan buscando alimento o parejas con las cuales reproducirse. La probabilidad de encuentro entre individuos reproductivos disminuye.

En ambos ejemplos, mientras más pequeña es una población, mayor su tasa de endogamia y menor la varianza genética, lo que reduce aún más el tamaño de las poblaciones. La probabilidad 
de extinción debido a fenómenos naturales como incendios y huracanes, que ocurren al azar es mayor y los organismos caen en un vórtice de extinción del que no se recuperan (PRIMACK, 2002). Este efecto, conocido como "efecto Allee", es considerado de extrema importancia en la conservación de especies raras o en peligro de extinción. En Colombia, varias especies pudieran hallarse en este caso, como son el tití cabeciblanco (Saguinus oedipus), el mono aullador (Alouatta seniculus), el tigrillo (Leopardus tigrinus) y el venado de cola blanca (Odocoileus virginianus), que han visto muy disminuido el tamaño efectivo de sus poblaciones debido fundamentalmente a la fragmentación de su hábitat por al cambio de uso de grandes extensiones de bosques para la agricultura y la ganadería (BOTERO et al. (2011). Sin embargo, solo Saguinus oedipus aparece reportada en peligro crítico (CR) por el MINISTERIO DE MEDIO AMBIENTE Y DESARROLLO SOSTENIBLE (2014).

Algunas conductas son sensibles a la perturbación (GONZÁLEZ-ZUARTH, 2015), como es el caso de la elección de la pareja cuando esto se basa en el dimorfismo sexual. La selección natural sexual es un tipo de selección natural que TRIVERS (1972) denominó intersexual o epigámica y que opera sobre los rasgos que le dan al individuo mayores posibilidades de ser elegido por un miembro del sexo opuesto. En muchas especies tales rasgos, como colorido, diseños y otros, se hacen tan conspicuos que, quien los posee, casi siempre el macho, estará en desventaja debido a que son mucho más fácilmente detectados por los depredadores. En esos casos se observa un sesgo en la relación macho hembra que puede ocasionar dificultades para la selección de la pareja e incluso que la hembra no se aparee con machos que presentan los mejores fenotipos. Se ha comprobado que cuando las hembras tienen pocas posibilidades de elección, disminuye su éxito reproductivo (SMITH y READ, 1992). MØLLER y LEGENDRE (2001) han planteado que la selección sexual puede generar un efecto Allee, sobre todo entre las especies con dimorfismo sexual, lo que traería las consecuencias que ya sabemos.

Otro ejemplo de conducta sensible a la perturbación es la supresión reproductiva (GONZÁLEZZUARTH, 2015). En este caso, numerosas especies suprimen parcial o totalmente la conducta reproductiva, quizás porque la alteración del ambiente suprime o altera los estímulos ambientales relacionados con tal actividad y esto trae como consecuencia la disminución del tamaño de la población efectiva con los conocidos efectos ya mencionados.

En cuanto a los impactos del hombre sobre conductas que afectan el tamaño poblacional efectivo (GONZÁLEZ-ZUARTH, 2015), se destacan la introducción de especies invasoras, muchas de las cuales son capaces de alterar el ambiente a un grado tal, que las estrategias conductuales de las especies nativas que eran exitosas, dejan de ser adaptativas; tienen además capacidad de invadir localidades realmente hostiles gracias a la cantidad de adaptaciones que poseen y por último, algunas presentan capacidad de hibridar con las especies nativas, lo que provoca con cierta frecuencia, la desaparición de estas últimas a causa de la introgresión. Este fenómeno no se encuentra suficientemente estudiado en nuestro país, a pesar de que son 88 las especies invasoras en territorio colombiano (FRANCO et al., 2011), las que aparecen en el "Plan Nacional para la Prevención, el Control y Manejo de las Especies Invasoras"del Ministerio del medio Ambiente y Desarrollo, donde se plantea que esta es la segunda amenaza sobre la diversidad biológica colombiana después del cambio climático global.

La cacería constituye otro impacto antropogénico importante sobre el tamaño poblacional efectivo de ciertas poblaciones porque elimina principalmente individuos de mayor talla, que generalmente son los de mayor edad y experiencia e incluso vistosidad $y$ esto hace que disminuya la probabilidad de que las hembras encuentren un macho que sea genéticamente superior y pasen sus genes a la próxima generación. Algo similar ocurre con la pesquería de grandes cardúmenes de peces que utilizan esa estrategia como defensa y para conseguir mejor el alimento, cuando se reducen esos grupos drásticamente, ya que el tamaño efectivo de las poblaciones se ve muy disminuido a su vez.

La conducta animal puede y debe aportar muchos elementos a la teoría de la conservación, pero también es capaz de aportar soluciones de tipo 
práctico a los problemas de conservación que aparecen en la naturaleza y en instalaciones donde permanecen especies silvestres. Cómo podría ayudarse a una especie de ave o mamífero en la que se ha disminuido su potencial reproductivo debido a la destrucción de su hábitat, si no es a través de la recuperación de ese hábitat y también propiciando las condiciones para su anidamiento o construcción de madrigueras. Pero esto último requiere el conocimiento exhaustivo de la conducta reproductiva de tales especies.

Si una población de una especie dada presenta un tamaño efectivo muy pequeño y se toma la medida de criarlos en cautiverio y posibilitar la repoblación en su hábitat más adelante, ¿cómo podría esto realizarse si no se conoce la conducta de alimentación, la reproductiva, e incluso la social?

La educación ambiental constituye una herramienta fundamental para la conservación de la diversidad biológica (CASSINI, 1999) y no se concibe su papel si no se acude a una base científica sólida que permita convencer y aún interesar a las personas de cualquier edad, sexo o condición social, en relación con el objeto de conservación. Normalmente los especialistas en esta tarea acuden a elementos de la conducta de las especies en peligro o sencillamente que caracterizan una localidad, buscando la motivación necesaria que promueva la conservación de tales localidades y/o especies. Mis alumnos de Biología de la Conservación estudian con profundidad la conducta de determinadas especies que son traficadas de forma ilegal en esta sub región del Caribe colombiano como la hicotea (Trachemys scripta callirostris) (DE LA OSSA et al., 2011) y el perezoso de tres dedos (Bradypus variegatus) (SAMPEDRO et al., 2011) para lograr la atención de los niños y padres acerca de la necesidad de detener este importante flagelo de la diversidad biológica.

Por último, la utilización de la conducta animal como indicador delestado de las áreas protegidas como parques nacionales, santuarios de fauna y otros, es también un elemento que debe considerarse como apoyo a la conservación de la diversidad biológica. Este aspecto no lo hemos hallado presente en la bibliografía disponible, pero está claro que las personas que trabajamos campo y acudimos casi a diario al escenario donde se llevan a cabo infinidad de conductas por parte de la fauna y sobre todo los que tienen la responsabilidad como especialistas de detectar problemas ambientales, utilizamos esos rasgos esenciales del comportamiento animal para diagnosticar el estado de tales sitios o detectar determinados fenómenos ambientales que no se aprecian a simpe vista.

Algunos ejemplos ayudarán. Personalmente he detectado conductas irregulares en primates como Alouatta seniculus(mono aullador) en algunas localidades de los Montes de María, donde se practica la minería legal e ilegal y las explosiones y deforestación en tales sitios hace que aparezcan individuos de ambos sexos y diferentes edades de esta especie sin contacto alguno con los grupos familiares que acostumbran constituir. Otro tanto parece ocurrir con la disminución del canto de las aves en época reproductiva en lugares donde es común escucharlas por cientos.

He observado muchas hicoteas del género Trachemys dentro del agua, en lagunas y pozos, en horas en que comúnmente utilizan para termorregular extendidas en playones o montadas sobre troncos secos y piedras (SAMPEDRO et al., 2002).Al medir la temperatura del agua he comprobado que alcanza valores altos y muy similares a la temperatura externa, por lo que he supuesto que han variado su conducta termorreguladora y ahorran así el gasto energético que implica subir a los sitios de termorregulación habituales.

La falta de cuidados parentales en estrategas $\mathrm{K}$, como la mayoría de los mamíferos y aves es algo que se detecta con cierta frecuencia en sitios alterados o donde se practica la caza.

La observación de peces acudiendo a la superficie en busca de oxígeno es prueba de la disminución de ese recurso por contaminación orgánica o inorgánica.

Por supuesto que el uso de la conducta como indicador demanda conocimientos sobre la historia natural y la etología de las especies, así como de la ecología de poblaciones y comunidades y sobre todo de la experiencia de campo y poder de observación del especialista, pero no cabe 
duda de que estos elementos unidos pueden resultar muy eficientes en la detección de disímiles problemas ambientales, lo que constituye el primer paso en las labores de conservación.La ecología de la conductaapunta en esa dirección, aplicar la teoría de esas disciplinas a la solución de los problemas ambientales.

El cambio climático global se siente con fuerza ya en nuestros días y las especies de fauna y nuestra misma especie deberemos adaptarnos a tales condiciones o no sobreviviremos. Quizás la nuestra sea una especie privilegiada por la gran variabilidad genética que ostenta. Sin embargo para muchas especies raras, debido a su pequeño número efectivo, la situación podría ser diferente. Será necesario que estudiemos y apliquemos todas las medidas necesarias para salvarlas y la ecología de la conducta deberá desempeñar un papel importante en esa misión.
Se requiere incrementar el conocimiento sobre la etología de las especies vulnerables, determinar su estado de conservación regional y nacional y aplicar las posibilidades que brinda la ecología de la conducta para anticipar las medidas necesarias de conservación que resulten verdaderamente efectivas. Otro campo del saber será sin dudas, el estudio de las nuevas estrategias adaptativas que irán desarrollando las especie para sobrevivir a este fenómeno ambiental.

Los biólogos del futuro podrán examinar la evolución de las adaptaciones actuales, corroborarán el papel de la conducta en la supervivencia de las especies, pero todos tendrán la misma tarea, conservar la vida del planeta.

\section{Referencias}

BEISSINGER, S.R., 1997. Integrating behavior into conservation biology: potentials and limitations. Págs. 23-47. EN: Clemmons, J.R.; Buchholz, R. (Eds.). Behavioral approaches to conservation in the wild Cambridge University Press. Cambridge.

BERGER-TAL, O.; POLAK, T.; ORON, A.; LUBIN, Y.; KOTLER, B.P.; SALTZ, D. 2011. Behavioral Ecology 22 (2):236239.

BEROVIDES, V. 1988. ¿Por qué los animales se comportan así?. Ministerio de Cultura, Editorial científico-Técnica, La Habana, Cuba.

BLUMSTEIN D.T.; FERNANDEZ-JURICIC, E. 2010. A primer of conservation behavior. Sinauer Associates. Sunderland (MA).

BOTERO, L.; DE LA OSSA, J. 2011. Fauna silvestre asociada a ganado vacuno doble propósito en sistema de silvopastoreo, Pinto, Magdalena, Colombia. Rev. MVZ Córdoba 16 (3):2733-2741.

CAMPBELL, D.T. 1975. Reintroducing Konrad Lorenz to Psychology. Págs. 106. En: Evans, R.I.; Lorenz, K. The Man and His Ideas. Harcourt Brace Jovanovich. New York.

CARO, T. 1998. Mating systems and conservation intervention. Págs. 270-276. En: Caro, T. (Ed.). Behavioral Ecology and Conservation Biology. Oxford University Press. New York.

CARRANZA, J. (Ed.). 2016. Etología adaptativa: El comportamiento como producto de la selección natural. Universidad de Extremadura y Córdoba. Cáceres, España..

CASSINI, M.H. 1999. Importancia de la etología en la Conservación. Etología, Etología 7:69-75. 
DE LA OSSA. V,J.: OLIVERO-GÓMEZ, V.; RUIZ, J.G. 2011. Utilización de quelonios de interés económico en el municipio de Caimito, Sucre, Colombia. Rev. ColombianaCienc. Anim. 3 (1): 3-14.

DARWIN, C. 1859. The origin of species by means of natural selection. Murray. London.

FESTA-BIANCHET, M.;APOLLONIO, M. 2003. Animal Behaviorand Wildlife Conservation. Island Press. Washington, D.C.

FRANCO, A.M.; BAPTISTE, M.P.; DÍAZ, J.M.; MONTOYA, M. 2011. Plan Nacional para la Prevención, el Control y Manejo de las Especies Introducidas, Trasplantadas e Invasoras: diagnóstico y listado preliminar de especies introducidas, trasplantadas e invasoras en Colombia. Ministerio deAmbiente y Desarrollo Sostenible. Bogotá, Colombia.

GONZALO ANDRADE, C,M. 2011. Estado del conocimiento de la biodiversidad en Colombia y sus amenazas. Consideraciones para fortalecer la interacción ciencia-política. Rev. Acad. Colomb. Cienc., XXXV (137):5491-507.

KREBS, J.R.; DAVIES, N.B. 1993. An Introduction to Behavioural Ecology. Third Edition.Blackwell Science Ltd. London, U.K.

KRUUK, H. 2003. Niko's Nature: The Life of Niko Tinbergen and His Science of Animal Behaviour. Oxford University Press. Oxford R.U.

MANNING, A. 1963. Selection for mating speed in Drosophila melanogaster based on the behaviour of one sex. Animal Behaviour 11 (1):1-220.

MINISTERIO DEL MEDIO AMBIENTE Y DESARROLLO SOSTENIBLE. 2014. Resolución 0192. Listado de las especies silvestres amenazadas de la diversidad biológica colombiana. Bogotá, Colombia.

MOLLER, A.P.; LEGENDRE, S. 2001. Allee effect, sexual selection and demographic stochasticity.Oikos 92:27-34.

PRIMACK, R.B. 2002. Essentials of Conservation Biology. (3a ed.. Sinauer Assoc., Inc. Sunderland, USA.

RUBENSTEIN, D. 1998. Behavioral ecology and conservation policy: on balancing science, applications, and advocacy. Págs.527-556. En: Caro, T. (Ed.). Behavioral ecology and conservation biology. Oxford University Press. Oxford.

SAMPEDRO, A.C.; CABEZA, K. 2009. Importancia de la conducta animal para el manejo productivo de la fauna silvestre y doméstica. Revista Colombiana de Ciencia Animal 2 (1):175-203.

SAMPEDRO, A.C.; AGUAS-MONTES, K.; JIMÉNEZ-PINEDA, D. 2011. Estado de conservación y caracterización del hábitat de Bradypus variegatus Schinz 1825 (Mammalia: Xenarthra) durante la época seca, en el departamento de Sucre, Colombia. Rev. Colombiana Cienc. Anim. 3 (1):15-34.

SHAPIRO, J.A. 2011. Evolution: A View from the 21st Century. FT Press Science. USA.

SMITH, R.L.; READ, B. 1992.Management parameters affecting the reproductive potential of captive, female black rhinoceros, Diceros bicornis. Zoo Biology 11:375-383.

SOLER, M. 2009. Adaptación del comportamiento: comprendiendo al animal humano, Síntesis. Granada, España.

TINBERGEN, N. 1951. The study of instinct. Clarendon Press. Oxford.

TINBERGEN, N. 1963.On aims and methods of ethology. Z. Tierpsychol. 20, 410-33.

TRIVERS, R. L. 1972. Parental investment and sexual selection. Campbell. pp. 136-179. ISBN 0-435-62157-2. 